\title{
BMJ Open Protocol for a prospective, longitudinal mixed-methods case study: supporting a Model of Care for Healthier Adolescents (The MoCHA study)
}

\author{
Claire Hayes, ${ }^{1,2}$ Victoria J Palmer, ${ }^{3}$ Magenta Simmons, ${ }^{4}$ Bridget Hamilton, ${ }^{5}$ \\ Christine Simons, ${ }^{1,2}$ Malcolm Hopwood ${ }^{1,2}$
}

To cite: Hayes C, Palmer VJ, Simmons M, et al. Protocol for a prospective, longitudinal mixed-methods case study: supporting a Model of Care for Healthier Adolescents (The MoCHA study). BMJ Open 2019;9:e025098. doi:10.1136/ bmjopen-2018-025098

- Prepublication history for this paper is available online. To view these files, please visit the journal online (http://dx.doi. org/10.1136/bmjopen-2018025098).

Received 2 July 2018

Revised 30 January 2019

Accepted 30 January 2019

Check for updates

(c) Author(s) (or their employer(s)) 2019. Re-use permitted under CC BY-NC. No commercial re-use. See rights and permissions. Published by BMJ.

${ }^{1}$ The Albert Road Clinic, Melbourne, Victoria, Australia 2Department of Psychiatry, Melbourne Medical School, The University of Melbourne, Melbourne, Victoria, Australia ${ }^{3}$ Department of General Practice, Melbourne Medical School, The University of Melbourne, Melbourne, Victoria, Australia ${ }^{4}$ Centre for Youth Mental Health, The University of Melbourne, Melbourne, Victoria, Australia ${ }^{5}$ Centre for Psychiatric Nursing, The University of Melbourne, Melbourne, Victoria, Australia

Correspondence to Ms Claire Hayes; c.hayes3@student.unimelb. edu.au

\section{ABSTRACT}

Introduction Improving mental healthcare for adolescents is a global policy priority. Despite demands for communitybased services, many adolescents require more intensive interventions, such as an inpatient admission. This is typically at a point of crisis, often accompanied by intense emotional dysregulation, impairment of coping function and impulsivity. However, limited evidence exists on how best to support this group while they are in inpatient care, aside from pharmacological treatments which have a limited role in adolescents. Little is known about the models of care $(\mathrm{MoC})$ offered in inpatient units, whether adolescents perceive these as helpful and the perspectives of caregivers and clinicians. Here, we describe a protocol which aims to explore and evaluate an inpatient MoC.

Methods and analysis We designed a longitudinal, mixed-methods, case study. The population consists of adolescents, caregivers and clinicians at a single inpatient unit in Melbourne, Australia. Standardised outcome measures, including semi- structured interviews, will be administered to adolescents at three time-points, T1 (admission), T2 (discharge) and T3 (6 months post discharge). Caregivers will also be interviewed at T1, T2 and T3. Clinicians will be interviewed once. The measures include: Life Problems Inventory, Quick Inventory of Depressive Symptomatology, Kessler Psychological Distress Scale and the Youth Self-Report. Health of the Nation Outcome Scales for Children and Adolescents will be collected at T1 and T2. Quantitative analysis will include descriptive statistics and paired t-tests summarising adolescents admitted to the unit, clinical characteristics and longitudinal data on symptomatology. Qualitative data will be analysed using both thematic and trajectory analysis. Data collection began in May 2017 and will cease with T3 interviews by October 2018 .

Ethics and dissemination

\section{INTRODUCTION}

Adolescence is a period involving the onset of behaviours and conditions that not only affect health during that time, but can also lead to disorders in adulthood. ${ }^{1}$ More than $50 \%$ of adult mental health disorders typically emerge before the age of 18 years. ${ }^{2} 3$
Strengths and limitations of this study

To the best of our knowledge, this is the first comprehensive case study design evaluating an adolescent inpatient model of care from the perspectives of adolescents, caregivers and clinicians.

- This study interviews adolescents and caregivers 6 months post-discharge from the inpatient unit, an advance on cross-sectional studies.

- The knowledge gained from this study has theoretical generalisability rather than statistical generalisability and may have great importance in allocating healthcare resources to benefit adolescents.

- The current study is too brief to show enduring outcomes; however it may provide important data for further evaluation of adolescents admitted to inpatient units and long-term outcomes.

- This study explores a single inpatient unit in Melbourne, Australia, thus limiting generalisability to other inpatient settings.

Despite efforts of clinicians and researchers worldwide, youth suicide statistics are a serious problem with rates continuing to rise on a global scale. ${ }^{4-6}$ These factors necessitate a focus on the access and care arrangements for adolescents in need of inpatient care. ${ }^{7}$ While the majority of adolescents with mental health problems continue to be cared for in the community, there are those who require more intensive treatment interventions such as inpatient care. ${ }^{89}$ The primary goals of inpatient care are containment of risk, containment of the dysfunctional distress responses, stabilisation of symptoms and the development of management and problem solving skills to ensure further supports can be facilitated and provided in community settings. ${ }^{10}{ }^{11}$ Limited evidence exists on how best to support this group while they are in inpatient care, aside from pharmacological treatments which have a limited role in adolescents. ${ }^{12}{ }^{13}$ Furthermore, little is known 
about adolescent inpatient units and, in particular, the models of care they provide.

According to Queensland Health, ${ }^{14}$ Model of Care (MoC) is a multifaceted concept, which broadly defines the way health services are delivered. A MoC should describe everything that is provided by the inpatient unit, such as the philosophical underpinnings, types of treatment, therapeutic interventions, staff and interdisciplinary team, support groups, design of the unit - physically and organisationally, treatment planning approaches and if it is person-centred and driven. Therefore, a MoC should include the fundamental characteristics and components of which the inpatient unit is comprised so that others may ascertain to what extent this MoC can be applied elsewhere. Instead, current studies tend to focus on the organisational structure, such as capacity, number of beds and length of stay, rather than components which matter most to the adolescents, caregivers and clinicians. ${ }^{9}$ For instance, few studies indicate whether their MoC is manualised. Such information would be of benefit to those aiming to evaluate or improve adolescent inpatient units.

The MoC concept varies internationally . In Australia, the term MoC is often referenced in terms of community-based models. This includes organisations such as Headspace, which originated in Australia. The Headspace model has been replicated in other countries such as Denmark, Israel and California, as well as Jigsaw in Ireland and Youthspace in the UK. ${ }^{15}$ All of the models are government-funded and governed by similar principles. This includes stigma-free early intervention services to support 12-25 year olds with emerging mental health disorders. ${ }^{15}$ Unlike community-based models, less is known about a desirable inpatient $\mathrm{MoC}$ for adolescents and how this might differ for public and/or private settings.

Indig et al examined when inpatient care is most effective, as well as the appropriate MoC for the treatment of children and adolescents with moderate-to-severe mental disorders. In their report of a rapid literature review, these researchers claimed that there was a range of models used for providing care to young people in inpatient setting. ${ }^{16}$ However, their portrayal of the MoC in each study did not provide sufficient details or descriptions for imitation and/or comparison of practices. Similarly, a recent systematic review found that adolescent inpatient unit studies were lacking in their ability to capture inpatient settings and the MoC. ${ }^{9}$ Following the review by Indig et al, table 1 was constructed to understand what we currently know about adolescent inpatient MoC. It has been divided into three levels, which are policy, organisational and individual levels.

Particularly in the last 10 years, adolescent studies which refer to the MoC predominantly discuss models outside the inpatient setting, such as models suggested as alternatives to inpatient care or nurses' proposed development of the MoC in which they practice. ${ }^{17-19}$ Other inpatient unit studies primarily focus on effectiveness, narrowly defined in terms of symptom stabilisation from admission to discharge, reporting effectiveness for the majority of adolescents. ${ }^{9}$ In addition to the methodological limitations, these studies fail to portray in any detail the MoC adopted from the adolescents' perspective, giving rise to inadequate reporting, gaps in understanding how a MoC is experienced and making the potential for comparisons and interpretation difficult. Therefore, the lived-experience perspective is essential, given the policy directives to involve the participation of consumers in the planning, design and evaluation of services. ${ }^{20}$

Overall, the term MoC term is adopted occasionally in adolescent inpatient literature, but does not appear to have a consistent definition and is poorly understood. Therefore, an examination of how adolescents understand and experience a MoC from the inpatient setting is essential and will assist to clarify what is required for a contemporary MoC. More importantly, a well-articulated MoC might support improvements in experiences of care and mental health outcomes for adolescents. This protocol describes a study to explore and understand an inpatient MoC for adolescents using a longitudinal mixed-methods

Table 1 Features of an inpatient model of care for adolescents

\begin{tabular}{|c|c|c|c|}
\hline & Policy level & Organisational level & Individual level \\
\hline $\begin{array}{l}\text { Who } \\
\text { Who should provide it? }\end{array}$ & $\begin{array}{l}\text { Crisis admissions/Not for crisis } \\
\text { admission }\end{array}$ & Multidisciplinary teams & $\begin{array}{l}\text { Accepted mental health } \\
\text { disorders } \\
\text { Criteria for exclusion } \\
\text { Accepted age range }\end{array}$ \\
\hline $\begin{array}{l}\text { Where } \\
\text { Where should it be provided? }\end{array}$ & $\begin{array}{l}\text { Location } \\
\text { Catchment area }\end{array}$ & & \\
\hline
\end{tabular}


case study design. It aims to address important gaps in previous studies, particularly in relation to reporting and from the perspectives of adolescents, caregivers and clinicians. This comprehensive approach to studying an adolescent inpatient MoC will lay a foundation for determining the effectiveness and future application of such models in both public and private settings.

\section{Aim and research questions}

The overarching aim of the Model of Care for Healthier Adolescents (MoCHA) study is to further the understanding of a current adolescent inpatient MoC for academic, policy and practice purposes and to support systematic work to improve mental health outcomes for young people. The longitudinal mixed-methods study will address the following aims:

1. To describe a current inpatient MoC for adolescents.

2. To explore the experiences of adolescents, caregivers and clinicians in relation to the MoC.

3. To evaluate the perceived helpfulness of the MoC on adolescent mental health, symptoms and quality of life (QoL) from the perspectives of adolescents, caregivers and clinicians.

Research Questions

1. What is the MoC?

2. What are the experiences of adolescents, caregivers and clinicians in relation to the MoC?

3. Do adolescents, caregivers and clinicians perceive the MoC to be helpful for mental health, symptoms and QoL?

\section{METHODS}

\section{Study design and population}

A prospective, longitudinal, mixed-methods, case study design was adopted to explore and evaluate an adolescent inpatient MoC. The inpatient unit to be investigated will serve as the single case in this study. This paper describes the protocol for the whole study.

The study population will consist of adolescents who are admitted to an inpatient unit, their caregivers and clinicians who work in the inpatient unit. Quantitative and qualitative data will be collected from adolescents at baseline (at admission to the inpatient unit, T1), at discharge from the inpatient unit (T2) and at 6 months post-discharge (T3) (May 2017-October 2018). Qualitative data will be collected from caregivers at all three time-points to gain in-depth knowledge on processes and experiences. Clinicians will be invited to participate in one interview and these will be conducted between December 2017 and July 2018. The combination of quantitative and qualitative data will be used to describe the MoC, evaluate effectiveness where possible, identify the areas for improvement and to strengthen insight in relation to participants' experiences of the MoC. Furthermore, it will inform the understanding and examination of the MoC and its interrelationship with the outcomes and experiences of adolescents.
While the quantitative and qualitative data will be analysed separately using appropriate methods, a complementary analysis of both data sets will seek to establish the key features regarding the current MoC. These key features will be guided and developed from MoC descriptions provided in previous studies in an effort to capture the model in its entirety. Table 1 will be used as a baseline for features used to describe the current adolescent inpatient MoC. This will also be used to help guide a MoC framework from certain elements identified in the experiences of adolescents, caregivers and clinicians. This will include the organisational structure of the unit, therapeutic content of the programme and interventions provided. A detailed description of the type of adolescents who are admitted to the unit will also be provided, such as primary diagnosis, age, sex and mental health history. In addition, routinely collected data will be employed where possible.

The experiences of adolescents and caregivers in terms of previous mental health services will be explored at T1, as well as engagement with professionals, precipitating factors leading to the current admission and experiences of symptoms. T2 and T3 data will explore the experiences of the inpatient admission, as well as discharge from the service and management of symptoms. All of these concepts and dynamics will contribute to the MoC description and the analysis of trajectories. This will allow the identification of key components of participants' experiences of inpatient admission and clarify what possible interventions could be of benefit to adolescents, caregivers and clinicians.

\section{Study setting}

The MoCHA study will be conducted at the Albert Road Clinic (ARC) in Melbourne, Australia. ARC is part of Ramsay Health Care, which provides private healthcare in the UK, Australia, France, Indonesia and Malaysia. The adolescent inpatient unit, known as 'Pathways' is part of an 80-bed private hospital setting, which has been operating since 1975. The current MoC being offered in the inpatient unit has been in place since 2006. It is an important setting to study, as adolescents are admitted on a voluntary basis. This study setting is particularly valuable, as the adolescents participate in the compulsory therapeutic group programmes, which are facilitated by members of the multidisciplinary team (MDT), Monday to Friday from 09:00 to 16:30. The single case study design will enable understanding of the MoC in greater depth; however not all elements will be translatable to other settings.

The inpatient unit has 10-12 beds and caters to adolescents between the ages of 12 and 22. The variability in 10-12 beds is based on the cohort at the time and how adolescents are engaging with each other. This is decided based on discussions with the MDT and the Medical Director of the unit. The unit sits alongside an adult inpatient unit and all staff work across the adult and adolescent population. All staff work is informed by Dialectical Behaviour Therapy principles and staff receive monthly 


\section{Box 1 Eligibility criteria}

Inclusion criteria
Persons 12-22 years.
Has a mental health disorder.
Receives inpatient treatment.
Participates in the inpatient programme.
Provides informed consent to participate in study.
Caregivers provide informed consent for their adolescent to
participate.
Exclusion criteria
Not able to complete questionnaires.

training. A weekly adolescent team meeting takes place, where all adolescents are reviewed, with psychiatrists and members of the MDT in attendance. A wide variety of mental health disorders are accepted, provided the individual is evaluated as having the capacity to participate in group work and has the ability to engage in the programme.

\section{Participant recruitment}

Participant recruitment began in May 2017 and data collection will continue until October 2018. The study will enrol three groups of participants: adolescents who have been admitted to the inpatient unit, their caregivers and a sub-set of clinicians who work on the inpatient unit. Adolescents and their caregivers will be invited by the first author to participate in the study on admission to the inpatient unit. They will be provided with participation information and consent forms. The information will provide details about the nature and purpose of the study, as well as any associated potential risks. The adolescents and caregivers will be asked to contact the first author if they are interested in participating in the study. Like the general population, the adolescent participants will be heterogeneous, for example, in terms of age, sex, as well as the type of mental disorders. Box 1 shows details of eligibility criteria for the adolescent group.

Clinicians working on the inpatient unit will be invited by the first author to participate in the study during brief allocated times between clinical handovers. Participant information and consent forms will be provided. Interviews will be arranged at a time that is most convenient for the adolescent and/or caregiver. Interviews with adolescents will occur in the evening in an office on a separate floor from the inpatient unit once the group programme is finished for the day. Interviews with clinicians and caregivers will be conducted at a more suitable time, depending on work hours.

\section{Sample size}

The 'Pathways' unit typically admits approximately 100 adolescents annually. In terms of recruiting for the qualitative component, an estimated uptake rate of $40 \%-50 \%$ is expected due to the longitudinal nature of data collection, equating to approximately $40-50$ adolescents and caregivers. Had the study not required longitudinal data, a higher response rate could be anticipated. Retention of participants over a long period of time is challenging due to various factors such as returning to school, relapse of mental illness and other family issues. ${ }^{21}$ However, given that data saturation (that is, repeated and shared themes across the interviewees) will likely occur prior to this stage, interviews will most likely cease once 10-12 full data sets at admission T1 (admission), T2 (discharge) and T3 (6 months post discharge) are available. ${ }^{22}$ Ryan and Bernard (23) claim data saturation depends on the complexity of data, investigator fatigue and number of analysts reviewing the data. ${ }^{23}$ Among the clinicians, it is anticipated that 10-12 interviews will suffice to capture the description of the MoC. ${ }^{22}$ In terms of the quantitative component and in order to capture a larger descriptive representative sample, adolescents who do not wish to participate in the qualitative component will be asked if demographic data can be obtained from their medical file, to represent a larger subset. The goal for recruitment for this phase is $77 .^{24}$ The anticipated sample size is based on the review of previous adolescent inpatient unit studies. ${ }^{9526}$ However, it is important to report that these previous studies demonstrate considerable variability in response rates and effect sizes. The anticipated sample size is based on a study within Australia using the same outcome measure. ${ }^{25}$

\section{Data collection}

The MoCHA study will provide multiple sources of evidence for the exploration of the MoC. This evidence will come from three main sources: demographic data, administered validated and standardised outcome measure questionnaires and face-to-face semi-structured interviews. Data will be collected from May 2017 to October 2018. The data recruitment strategy for this study is presented in figure 1 .

\section{Demographic data}

Demographic variables will be recorded in relation to the following: age, gender, ethnicity, postcode, socio-economic status, length of hospital admission, previous hospital admissions, family and education history.

\section{Questionnaires}

Prior to each interview, adolescents will be asked to complete the outcome measure questionnaires. These questionnaires will allow the research team to observe whether aspects such as QoL, anxiety, depression, emotional regulation and impulsivity change over time. Furthermore, these questionnaires will be used to help answer aims 2 and 3 of this study

1. Life Problems Inventory (LPI): the 60-item consistent and validated self-report instrument was developed to assess emotion dysregulation, impulsivity, interpersonal chaos and confusion about self ${ }^{27}$ (T1, T2 and T3).

2. Quick Inventory of Depressive Symptomatology (QIDSSR): the 16-item self-report instrument was developed 


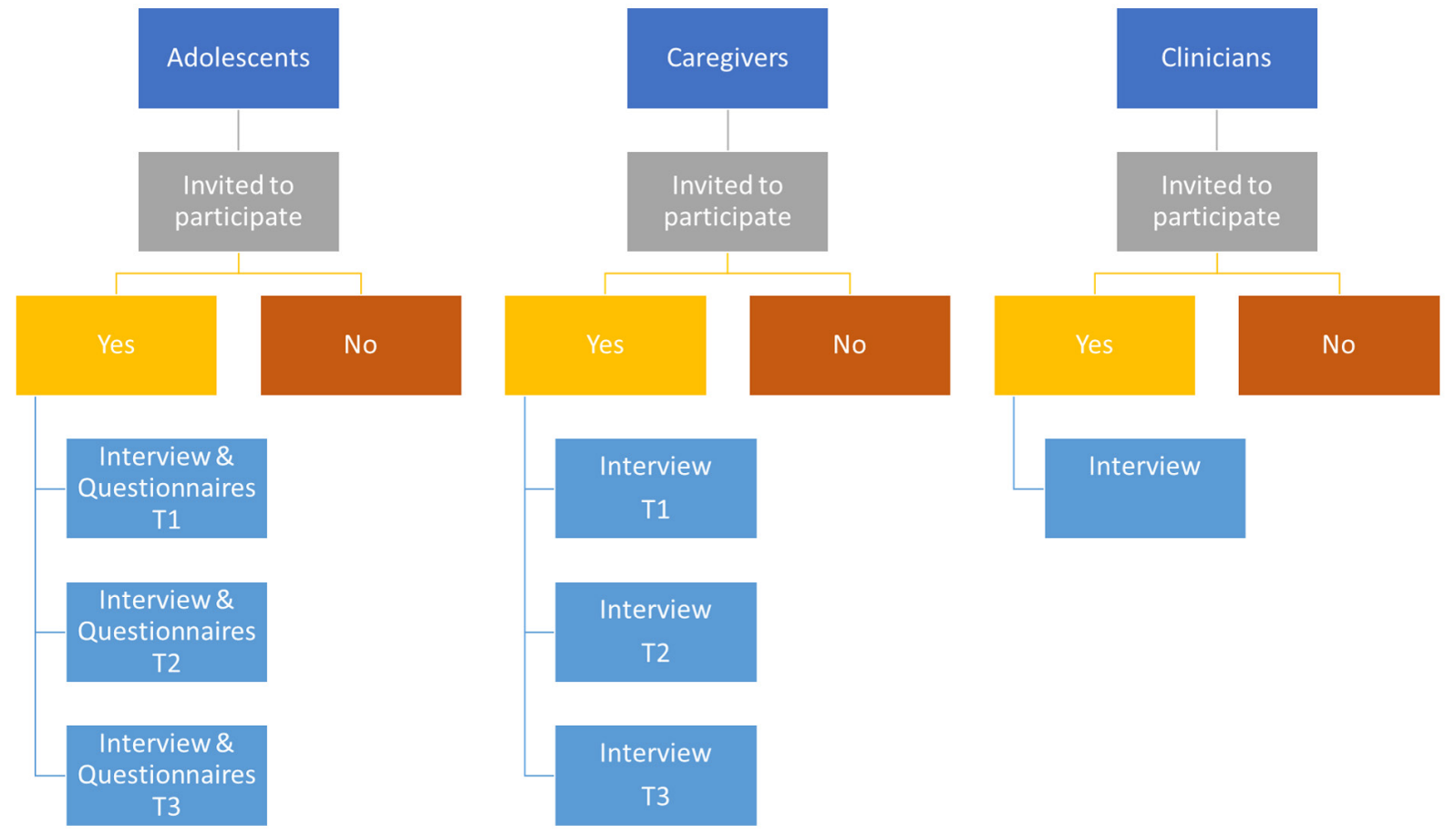

Figure 1 Flow diagram of recruitment strategy (MoCHA, Model of Care for Healthier Adolescents).

to assess depressive symptomatology. The instrument has demonstrated satisfactory validity and reliability for adolescent populations $^{28}$ (T1, T2 and T3).

3. Kessler Psychological Distress Scale (K-10): a 10-item self-report measure of psychological distress, which has been shown to be highly correlated with the presence of depressive or anxiety disorders ${ }^{29}$ (T1, T2 and T3).

4. Youth Self-Report (YSR): widely used 112-item measure self-report used to assess emotional and behavioural problems $^{30}$ (T1, T2 and T3).

5. HoNOSCA-SR (Health of the Nation Outcome Scales for Children and Adolescents): a 13-item self-report measure for general health and social functioning ${ }^{31}$ (T1 and T2).

These measures were selected to provide an objective standardised measure of changes over time. The LPI measures emotional dysregulation, impulsivity, interpersonal chaos and confusion about self. The QIDS-SR measures depressive symptomatology, while K-10 measures psychological distress. The YSR measures problem behaviours, while HoNOSCA measures general health and social functioning. All measures have been widely used in adolescent settings and have shown good reliability. The first author will assist participants to complete the questionnaires, as required, which should take up to 30 to 40 minutes and this will occur prior to the interview to minimise the influence of topics discussed on written questionnaire completion.

\section{Semi-structured interviews}

Semi-structured face-to-face interviews will be conducted on a separate level to the inpatient unit at ARC for confidentiality purposes. Interviews will take approximately
30 to 60 minutes for clinicians and 30 to 90 minutes for each interview with adolescents and caregivers.

Semi-structured interview schedules were developed based on qualitative methodology guidelines. ${ }^{32}$ These schedules are designed to be flexible with non-directive questions. During the development stage of the adolescent and caregiver semi-structured interview schedules, regular consultation will be sought from the medical director of the inpatient unit. Throughout this process, the 'I don't know' responses will be considered, which often occur when interviewing adolescents. The format of the schedules was chosen to allow the interviewer to remain flexible and follow-up on potential themes emerging at each interview. The adolescent and caregiver interview schedules are similar, exploring the adolescent's background and previous mental health treatments, mood, anxiety, QoL and relationships. The researchers will pay close attention to how adolescents are managing their mental health symptoms at T1, T2 and T3. The adolescent and caregiver interview schedules will be used flexibly, drawing on examples given at previous interviews. Open and non-directive questions will be asked as much as possible to limit the influence the interviewer has on the answers given by participants. At times, more direct questions and follow-up probes may be required to clarify themes that are emerging and to ensure that rich descriptions are obtained.

Interviews with adolescents, caregivers and clinicians

Consenting adolescents and caregivers will be interviewed separately and interviews will occur at T1 (admission), T2 (discharge) and T3 (6 months post discharge). The interviews with clinicians will aim to capture the clinician's views 


\section{Box 2 Semi-structured interview schedules}

Adolescents
Views on previous mental health services and interventions.
Relationships with mental health professionals.
Precipitating factors leading to inpatient admission.
Experience of mental health symptoms and quality of life (QoL).
Management of and coping with symptoms.
Expectations of the inpatient model of care (MoC).
Experience of inpatient admission and perceived helpfulness.
Caregivers
Views on previous mental health services and interventions.
Relationships with mental health professionals.
Precipitating factors leading to inpatient admission.
Experience of symptoms and QoL.
Management of and coping with symptoms.
Exptations of the inpatient MoC.
Elinicians
Most important features of the MoC.
Benefits of an inpatient MoC.

of the inpatient MoC and their perspectives of the work which they pursue every day. These interviews also aim to capture the various therapeutic interventions which occur in an inpatient unit. See box 2 for the semi-structured interview schedules to be used in this study. The interview schedules were also developed in consultation with the medical director of the inpatient unit.

\section{Transcription}

All interviews will be recorded on an audio device (with consent obtained from participants) and transcribed professionally. All transcripts will be checked against the audio files for accuracy and any references which might identify the participants will be removed. The same process will occur for clinicians due to the small number working in the inpatient unit. Transcripts will be stored in a password protected database and will be coded using NVivo V.10 (qualitative data management software). ${ }^{33}$

\section{Data analysis}

The data from the sub-set of clinicians will be utilised to describe the MoC and analysed using a thematic approach. The case will be formulated from the researcher's own knowledge of the research setting, discussions with key stakeholders such as the medical director of the inpatient unit, as well as admitting psychiatrists. The thematic analysis approach will be adopted to derive major and minor themes as guided by Braun and Clarke. ${ }^{34}$ Longitudinal data from adolescents and their caregivers will be used to explore their experiences of the MoC. Furthermore, it will be adopted to evaluate the perceived effectiveness of the MoC on adolescent mental health, symptoms, QoL and recovery. The longitudinal data will be analysed using a trajectory approach to explore the experiences and perceived helpfulness. The questionnaire outcome data will be incorporated to understand and answer some questions related to the MoC such as: what is working, why, how, for whom does it work best, for whom it does not it work and why?

\section{Qualitative component}

Qualitative interview data from adolescents and caregivers will be used in two ways. First, to explore thematically the experiences of the MoC and interactions with different elements and areas for improvement and strengthening. Thematic analysis was chosen as opposed to content analysis due to the semi-structured nature of the interview schedules and for allowing unexpected themes to arise. Second, it will be used longitudinally to explore and understand, from a trajectory-based approach, the experiences, as they relate with outcomes and effectiveness. Trajectory analysis focuses on changes over time and is often recommended to understand healthcare processes. ${ }^{35}$ Grossoehme and Lipstein suggest using time-ordered, sequential matrices, as time-ordered displays can preserve 'chronological flow' and permit understanding of what led to what. ${ }^{36}$

Codes will be identified from the interviews, clustered and formulated into themes. The data will be organised within matrices with one matrix per unit of analysis, such as the adolescent, caregiver, the adolescent and his or her caregiver or other groupings. Codes from participants, such as adolescents, will be identified via labelling through the use of colour coding. This will be conducted to create a visual overview of the data. This first set of matrices will be organised with themes along the Y-axis and time along the $\mathrm{X}$-axis (see table 2 for example). Once the coding has been completed, longitudinal analysis will begin. This step will focus on how the data changed or did not change over time. To organise the findings, another matrix will be needed. The Y-axis will again be organised by themes while the $\mathrm{X}$-axis will be organised according to the primary units of analysis (see table 3 for example). As

\begin{tabular}{llll}
\hline Table 2 & Sample adolescent matrix & & \\
\hline Themes & T1 & T2 & T3 \\
\hline $\begin{array}{l}\text { Theme A } \\
\text { (eg, acceptance) }\end{array}$ & $\begin{array}{l}\text { Lots of worry about not being } \\
\text { accepted by their family }\end{array}$ & $\begin{array}{l}\text { Feeling worried that other } \\
\text { adolescents will not accept } \\
\text { them }\end{array}$ & $\begin{array}{l}\text { Less worry about acceptance. Feel } \\
\text { accepted by other adolescents }\end{array}$ \\
$\begin{array}{ll}\text { Theme B (eg, hope) } \\
\text { Worried that there is no hope for } \\
\text { the future. }\end{array}$ & $\begin{array}{l}\text { Less worry about the future. } \\
\text { Feeling hopeful about the future and } \\
\text { making career plans }\end{array}$ \\
\hline
\end{tabular}


Table 3 Sample adolescent longitudinal analysis matrix

\begin{tabular}{|c|c|c|c|}
\hline Themes & T1 & T2 & T3 \\
\hline $\begin{array}{l}\text { Theme } A \text { (eg, change in } \\
\text { acceptance over time) }\end{array}$ & $\begin{array}{l}\text { Change from being worried about acceptance within the family to } \\
\text { acceptance by peers. Moved towards acceptance after admission }\end{array}$ & $\begin{array}{l}\text { Idea from } \\
\text { adolescent }\end{array}$ & $\begin{array}{l}\text { Idea from } \\
\text { adolescent }\end{array}$ \\
\hline
\end{tabular}

coding for the second matrix progresses, new conceptual groups might be needed as the original groupings will likely focus on cross-sectional concepts and new time-related concepts will emerge during coding. Data analysis will be conducted from the second matrix in which the codes will be focused on time. ${ }^{35}$

Although the first author will undertake the primary coding and analysis, the analysis process will be discussed with supervisors at regular meetings. In addition, a smaller subsample will be double coded during the initial stages of the analysis. However, this will not take place during the trajectory analysis stage, as this would be too difficult and likely interfere with the analysis process. The results of all analyses will be discussed by the entire supervisory research team. The data analysis process will adhere to the quality criteria described by Lincoln and Guba. ${ }^{37}$ This is to ensure trustworthiness and rigour in terms of credibility, transferability, dependability and confirmability. Although member checking will not be adopted, the first author will regularly clarify participant responses throughout the interviews to minimise the risk of misinterpretation.

\section{Quantitative component}

The demographic information collected at baseline (T1) will be tabled. Categorical variables will be reported as raw numbers and percentages. Reports of continuous variables will include mean, median, range and standard deviation. The statistical analyses will be performed using the Statistical Package for Social Sciences (SPSS) software (Version 25). Paired t-tests will be conducted with 0.05 significance level. Paired-sample t-tests will be conducted to evaluate whether there is a significant difference between T1, T2 and T3 on the LPI, K-10, QID-SR, YSR and HoNOSCA mean score ratings. Pearson product-moment correlation coefficients will be calculated to investigate associations between outcome measure score disparity/ admission scores between T1, T2 and T3. Correlations will be performed separately for each diagnostic category and for all participants collectively to identify associations between components of the MoC and outcomes regarding symptoms and QoL. Finally, repeated measures analysis of variance will be conducted, along with subsequent paired t-tests, to analyse differences between specific time points.

\section{Data management and monitoring}

Throughout this study, measures will be implemented to manage data and protect participants' identity. Following the interview process, audio tapes will be transferred to a password-protected computer and returned to a secure location. Once the audio tapes are transferred, the interview will be removed from the device. Field notes and transcripts will also be stored in a secure location. Codes will be allocated to participants, maintaining anonymity throughout the study. Participants will also be informed that publications or any other disseminated data will not include entire interview transcripts in the event of being identified. Instead, themes will emerge from the data which will be supported by quotations from the transcripts. All data will be stored in accordance with the Data Protection Act.

\section{Limitations}

There are limitations which pertain to this study. This study will explore a MoC at one inpatient unit in Melbourne, Australia. Therefore, results may not be generalisable to other inpatient units. Evaluations of adolescent inpatient units are not always subjected to rigorous research designs, such as the absence of randomised controlled trials. However, this study will add to evidence-based healthcare by employing research methods which help to describe in detail and understand an inpatient MoC. The anticipated sample size is small. However, this study seeks to explore analytical generalisability rather than statistical generalisability. All participants will be approached, but there is no control group and participants in this study will not be randomly assigned; so there is potential for selection bias. Finally, future hospital admissions will be not assessed after T2 unless discussed by participants in the interview.

\section{CONCLUSION}

Adolescence is an important time for early intervention, with the aim to minimise the risk of further deterioration and ultimately improve both short-term and long-term outcomes. The novel design of this study, drawing on qualitative and quantitative approaches has the potential to produce important advances in terms of what we know about inpatient care. Better articulation of what a MoC is comprised and the ability to describe these elements may see greater implementation of a clear MoC with outcomes. It is anticipated that the longitudinal mixed-methods research approach will enable a richer understanding and exploration of the trajectories of participants. This study will use the knowledge gained from adolescents and their caregivers who experience inpatient admission to inform other settings in terms of key components which are perceived to be most or least helpful. The findings 
will provide new information to inform relevant stakeholders when developing or implementing a similar service. The findings of this study will also address an important research gap, by providing what appears to be the only attempt to comprehensively explore and understand an inpatient MoC for adolescents, to guide and direct future models.

\section{Patient and public involvement statement}

The research questions and outcome measures were chosen based on discussions with key stakeholders of adolescent mental health services as well as investigations of the available adolescent inpatient outcome studies. Adolescent discussions took place to establish their priorities in terms of perceived improvement in areas such as QoL. This also occurred with clinicians who work in the inpatient unit. The research design was presented to adolescents at a youth mental health conference, ensuring their participation and input in the design. Adolescents will not be involved in the recruitment stages of the study. However, we endeavour to disseminate findings to adolescents, their families and clinicians at knowledge exchange workshops and conferences, locally and internationally.

\section{Ethics and dissemination}

The ethical approval process involved submitting a detailed research proposal to the committee. Following the committee's review of the research proposal, a meeting took place. Potentially harmful aspects of the study were raised and discussed by members of the committee and attendees. The issues were primarily based on the dual researcher/clinician role and research with vulnerable adolescents. Issues regarding anonymity of participants throughout the study also arose and were discussed.

Once the issues were discussed, a conscious effort was made to assure the ethics committee that appropriate measures would be put in place to protect participants. Such measures included ensuring data would be securely managed, interviews would be held in a private location with minimal distractions and entire interview transcripts would not be published. It was also decided that a clinician not working with the adolescent participants would obtain informed consent for the duration of the study. The separation of the clinician and researcher role was discussed and how this would be maintained throughout the study.

Ethical approval took several months to ensure any unethical issues were avoided. The research study was ethically approved by both committees on the 22 April 2017. Written informed consent will be obtained from all participants in the study once they have read and understood information regarding the study. Consent will also be required from a caregiver of the adolescent, should he/she wish to participate.

Findings from this study will be submitted for publication in peer-reviewed journals, and presented at national and international conferences relating to adolescent health services and quality improvement. A report will be compiled and presented to clinical staff and management.
The summative evaluation report will be presented to Ramsay Health Care. A knowledge exchange workshop will be facilitated to present findings to key stakeholders such as Ramsay Health Care and adolescent participants to discuss ways in which findings can be implemented and improved in the future. Social media will also be used as a platform to disseminate findings.

Acknowledgements We acknowledge the support and guidance from adolescents who were consulted in the development of the research design, as well as mental health stakeholders. We would also like to acknowledge the financial support from the Ramsay Health Care Ella Lowe Scholarship. Finally, we acknowledge the support and contribution of clinicians at the adolescent inpatient unit in Albert Road Clinic.

Contributors $\mathrm{CH}, \mathrm{VJP}, \mathrm{MS}, \mathrm{BH}, \mathrm{CS}$ and $\mathrm{MH}$ designed the protocol for this study. All authors supervised the design and revised the manuscript. All authors read and approved the final version of the manuscript.

Funding This research was supported by the Ramsay Health Care Ella Lowe Scholarship.

Disclaimer The study was partially funded by Ramsay Health Care. However, the funders had no role in the design of the study, data collection and analysis. Furthermore, funders were not involved in the decision to publish or preparation of the manuscript.

Competing interests None declared.

Patient consent for publication Obtained.

Ethics approval Ethical approval was sought and granted by the Ramsay Health Care Ethics Committee (protocol number EC00242). Once approval was granted, the study was registered with the University of Melbourne's ethics committee.

Provenance and peer review Not commissioned; externally peer reviewed.

Open access This is an open access article distributed in accordance with the Creative Commons Attribution Non Commercial (CC BY-NC 4.0) license, which permits others to distribute, remix, adapt, build upon this work non-commercially, and license their derivative works on different terms, provided the original work is properly cited, appropriate credit is given, any changes made indicated, and the use is non-commercial. See: http://creativecommons.org/licenses/by-nc/4.0/.

\section{REFERENCES}

1. Das JK, Salam RA, Lassi ZS, et al. Interventions for adolescent mental health: an overview of systematic reviews. J Adolesc Health 2016;59:S49-S60.

2. Jones PB. Adult mental health disorders and their age at onset. 2013;s5.

3. Kessler RC, Amminger GP, Aguilar-Gaxiola S, et al. Age on onset of mental disorders: a review of recent literature. 2007;359.

4. Australian Bureau of Statistics. Causes of Death, Australia, 2016, Canberra 2017 [26/11/2017]. Available: http://www.abs.gov.au/ ausstats/abs@.nsf/Lookup/by\%20Subject/3303.0 2016 Main\% 20Features Australia's\%20leading\%20causes\%20of\%20death, \%20 2016 3

5. Samaritans. Suicide Statistics Report 2017. United Kingdom, 2017 Available. https://www.samaritans.org/sites/default/files/kcfinder/ files/Suicide_statistics_report_2017_Final.pdf (cited 26 Nov 2017).

6. WHO. Suicide. In: WHO, ed. Geneva, 2018.

7. New South Wales Health. Children and Adolescents with Mental Health Problems Requiring Inpatient Care. In: Government N, ed. New South Wales, Australia, 2011.

8. Delaney KR. Special edition on inpatient treatment: A new annual tradition. J Child Adolesc Psychiatr Nurs 2017;30:160-1.

9. Hayes C, Simmons M, Simons C, et al. Evaluating effectiveness in adolescent mental health inpatient units: a systematic review. Int $J$ Ment Health Nurs 2018;27:498-513.

10. Hanssen-Bauer K, Heyerdahl S, Hatling T, et al. Admissions to acute adolescent psychiatric units: a prospective study of clinical severity and outcome. Int J Ment Health Syst 2011;5:1-11.

11. Tharayil $P$, James $S$, Morgan R, et al. Examining outcomes of acute psychiatric hospitalization among children. 2012;205.

12. Zhou X, Hetrick SE, Cuijpers $P$, et al. Comparative efficacy and acceptability of psychotherapies for depression in children and 
adolescents: A systematic review and network meta-analysis, 2015:207-22.

13. Caspi A, Houts RM, Belsky DW, et al. The p Factor: One general psychopathology factor in the structure of psychiatric disorders? Clin Psychol Sci 2014;2:119-37.

14. Health Q. Changing Models of Care Framework. Queensland, Australia: Queensland Health, 2000.

15. McGorry PD, Mei C. Early intervention in youth mental health: progress and future directions. Evid Based Ment Health 2018;21:182-4

16. Indig D, Gear C, York A. The role of inpatient care for children and adolescents with moderate-to-severe mental disorders: an Evidence Check rapid review brokered by the Sax Institute: NSW Ministry of Health, 2017. http://www.saxinstitute.org.au

17. Adrian N, Smith JG. Occupied bed days a redundant currency? An evaluation of the first 10 years of an integrated model of care for mentally ill adolescents. Clin Child Psychol Psychiatry 2015;20:458-71.

18. Foster K, Isobel S. Towards relational recovery: Nurses' practices with consumers and families with dependent children in mental health inpatient units. Int J Ment Health Nurs 2018;27:727-36.

19. Shepperd S, Doll H, Gowers S, et al. Alternatives to inpatient mental health care for children and young people. Cochrane Database Syst Rev 2009;2:CD006410.

20. United Nations. Report of the United Nations High Commissioner for Human Rights. In: United Nations, ed. 2017:13.

21. Polit DF, Beck CT. Nursing Research: Genrating and Assessing Evidence for Nursing Practice. 8th edn. Philadelphia: Wolters Kluwer/ Lippincott Williams \& Wilken, 2008.

22. Guest G, Bunce A, Johnson L. How Many Interviews Are Enough?: An Experiment with Data Saturation and Variability. Field Methods 2006;18:59-82.

23. Ryan GW, Bernard HR. Techniques to Identify Themes. Field methods 2003:15:85-109.

24. Faber J, Fonseca LM. How sample size influences research outcomes. Dental Press J Orthod 2014;19:27-9.
25. Burgess P, Pirkis J, Coombs T. Modelling candidate effectiveness indicators for mental health services. Aust N Z J Psychiatry 2009;43:531-8.

26. Hanssen-Bauer K, Heyerdahl S, Hatling T, et al. Admissions to acute adolescent psychiatric units: a prospective study of clinical severity and outcome. Int J Ment Health Syst 2011;5:1.

27. Rathus JH, Wagner D, Miller AL. Psychometric Evaluation of the Life Problems Inventory, a Measure of Borderline Personality Features in Adolescents. J Psychol Psychother 2015;5:198.

28. Bernstein IH, Rush AJ, Trivedi MH, et al. Psychometric properties of the Quick Inventory of Depressive Symptomatology in adolescents. Int J Methods Psychiatr Res 2010;19:185-94.

29. Chan SM, Fung TCT. Reliability and validity of K10 and K6 in screening depressive symptoms in Hong Kong adolescents. 2014;75.

30. Ebesutani C, Bernstein A, Martinez JI, et al. The youth self report: applicability and validity across younger and older youths. J Clin Child Adolesc Psychol 2011;40:338-46.

31. Urben S, Baier V, Mantzouranis G, et al. The French adaptation of the Health of the Nation Outcome Scale for Children and Adolescents Self-Rated Form (F-HoNOSCA-SR): validation and clinical routine use. Psychiatry Res 2014;218:229-35.

32. Smith JA, Larkin M, Flowers P. Interpretative phenomenological analysis: theory, method and research. Los Angeles; London: SAGE 2009;2009.

33. QSR International Pty Ltd. NVivo qualitative data analysis Software. In: $10 \mathrm{~V}$, ed. 2012.

34. Braun V, Clarke V. Using thematic analysis in psychology. Qual Res Psychol 2006;3:77-101.

35. Grossoehme D, Lipstein E. Analyzing longitudinal qualitative data: the application of trajectory and recurrent cross-sectional approaches. BMC Res Notes 2016;9:136.

36. Miles M, Huberman A. Qualitative data analysis: an expanded sourcebook. 2 edn. Thousand Oaks: Sage Publications, 1994

37. Lincoln YS, Guba Y, Guba EG. Publishing S. Naturalistic Inquiry: SAGE Publications, 1985. 